Revista de Investigación de Física 23(2), (Jul-Dic 2020)

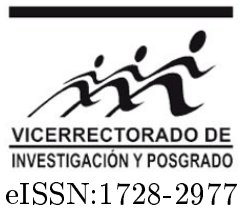

\title{
Estimación de estadíos estacionales a partir de parámetros climáticos medidos en la estación meteorológica de la microcuenca Apacheta, Región Ayacucho, 2000 al 2018
}

\author{
Wilmer Moncada ${ }^{* 1}$, Bram Willems² y Joel Rojas ${ }^{3}$ \\ ${ }^{1}$ Universidad Nacional San Cristóbal de Huamanga, Ayacucho, Perú \\ ${ }^{2}$ Centro de Competencia del Agua, Lima, Perú \\ ${ }^{3}$ Universidad Nacional Mayor de San Marcos, Lima, Perú
}

Recibido 07 abril 2020 - Aceptado 29 mayo 2020

\begin{abstract}
Resumen
La estacionalidad de la precipitación, temperatura máxima y mínima del aire, humedad relativa, velocidad del viento y radiación solar, impactan en el estado ecológico de la microcuenca Apacheta. El objetivo es estimar los estadíos estacionales a partir de parámetros climáticos medidos en la estación meteorológica de la microcuenca Apacheta, en base a la selección de intervalos de valores de precipitación. El estadío lluvioso comprende los meses de enero, febrero, marzo y diciembre, su precipitación es de $145.96 \mathrm{~mm}$ con temperatura máxima del aire de $12.3{ }^{\circ} \mathrm{C}$, temperatura mínima del aire de $1.55{ }^{\circ} \mathrm{C}$, humedad relativa de $74.04 \%$, velocidad del viento de $2.6 \mathrm{~m} / \mathrm{s}$ y radiación solar de 527.13 Ly. El estadío intermedio en abril, setiembre, octubre y noviembre, con precipitación de $51.89 \mathrm{~mm}$ con temperatura máxima del aire de $13.38{ }^{\circ} \mathrm{C}$, temperatura mínima del aire de $-0.2{ }^{\circ} \mathrm{C}$, humedad relativa de $69.76 \%$, velocidad del viento de $2.96 \mathrm{~m} / \mathrm{s}$ y radiación solar de $552.37 \mathrm{Ly}$. El estadío seco en mayo, junio, julio y agosto, con precipitación de $15.41 \mathrm{~mm}$, temperatura máxima del aire de $12.51{ }^{\circ} \mathrm{C}$, temperatura mínima del aire de $-2.4{ }^{\circ} \mathrm{C}$, humedad relativa de $67.49 \%$, velocidad del viento de $3.16 \mathrm{~m} / \mathrm{s}$ y radiación solar de 463.79 Ly.
\end{abstract}

Palabras clave: Precipitación, temperatura máxima del aire, temperatura mínima del aire, humedad relativa, radiación solar.

\section{Estimation of seasonal stages based on climate parameters measured at the Apacheta micro-basin weather station, Ayacucho Region, 2000 to 2018}

\begin{abstract}
The seasonality of rainfall, maximum and minimum air temperature, relative humidity, wind speed and solar radiation impact the ecological status of the Apacheta micro-basin. The objective is to estimate the seasonal stages from climatic parameters measured at the Apacheta micro-basin weather station, based on the selection of intervals of precipitation values. The rainy season includes the months of January, February, March and December, its precipitation is $145.96 \mathrm{~mm}$ with maximum air temperature of $12.3{ }^{\circ} \mathrm{C}$, minimum air temperature of $1.55{ }^{\circ} \mathrm{C}$, relative humidity of $74.04 \%$, wind speed of $2.6 \mathrm{~m} / \mathrm{s}$ and solar radiation of $527.13 \mathrm{Ly}$. The intermediate stage in April, September, October and November, with precipitation of $51.89 \mathrm{~mm}$ with maximum air temperature of $13.38{ }^{\circ} \mathrm{C}$, minimum air temperature of $-0.2{ }^{\circ} \mathrm{C}$, relative humidity of $69.76 \%$, wind speed of $2.96 \mathrm{~m} / \mathrm{s}$ and solar radiation of 552.37 Ly. The dry stage in May, June, July and August, with precipitation of $15.41 \mathrm{~mm}$, maximum air temperature of $12.51{ }^{\circ} \mathrm{C}$, minimum air temperature of $-2.4{ }^{\circ} \mathrm{C}$, relative humidity of $67.49 \%$, wind speed of $3.16 \mathrm{~m} / \mathrm{s}$ and solar radiation of $463.79 \mathrm{Ly}$.
\end{abstract}

Keywords: Precipitation, maximum air temperature, minimum air temperature, relative humidity, solar radiation.

${ }^{*}$ wilmer.moncada@unsch.edu.pe 


\section{Introducción}

La microcuenca Apacheta, de la Región Ayacucho, no es ajena a la influencia del cambio climático, el deshielo de sus nevados, el incremento de la temperatura del aire, la disminución de la precipitación, lo cual de alguna manera impacta en sus ecosistemas y por consiguiente al almacenamiento de agua en el reservorio Cuchoquesera que sirve para el consumo poblacional de la ciudad de Huamanga e irrigación de áreas agrícolas en la cuenca Cachi (Moncada et al., 2015). Actualmente el clima estacional de la microcuenca Apacheta se encuentra sujeta a tres estadíos estacionales, los mismos que son influenciados por la precipitación, la temperatura máxima y mínima del aire, la velocidad del aire, la humedad relativa y la radiación solar. Así como en algunos países como México las predicciones del cambio climático han establecido que en general la precipitación disminuye y la temperatura ambiente aumenta. Por ello, a través de enfoques como el primero de carácter estadístico para detectar tendencia o cambios en la media, que significa pérdida de homogeneidad en los registros de datos analizados; el segundo de tipo cuantitativo consistente en evaluar el cociente entre la precipitación media reciente y la de largo plazo para observar si se aparta de la unidad. Estimándose una precipitación media estable, se encontró que no existe evidencia estadística general de tendencia, pues únicamente dos registros mostraron un comportamiento decreciente; sin embargo, la lluvia anual en ciertas zonas se está reduciendo tal como lo detecta el cociente entre la lluvia media de los últimos 15 años y la de largo plazo, cuya moda de 27 valores es 0.98 (Campos, 2015). En otras zonas de los Andes, como las zonas cafetaleras de Colombia, la variación temporal de la temperatura es mínima, en su mayor parte la variabilidad de este elemento es espacial, atribuible casi exclusivamente a los cambios en altura sobre el nivel del mar. El Niño Oscilación del Sur y el Cambio Climático, inciden sobre esta variable climática (Pérez et al., 2016).

Por otro lado, la radiación solar suele darse con mayor intensidad en ciertas estaciones del año y suele ser uno de los principales factores etiopatogénico de las formas más frecuentes del cáncer de piel, por lo que es más frecuente en meses estivales llegando alcanzar el índice UV valores máximos de 7 a 8 y de 1 a 2 en meses de invierno (Aguilera et al., 2004). La variabilidad de la humedad atmosférica relativa y la concentración de partículas de aerosol en diferentes estadíos estacionales provocan formación de turbidez, donde las reacciones físicas y químicas en las superficies de las partículas conducen a variaciones en el tamaño de las partículas.

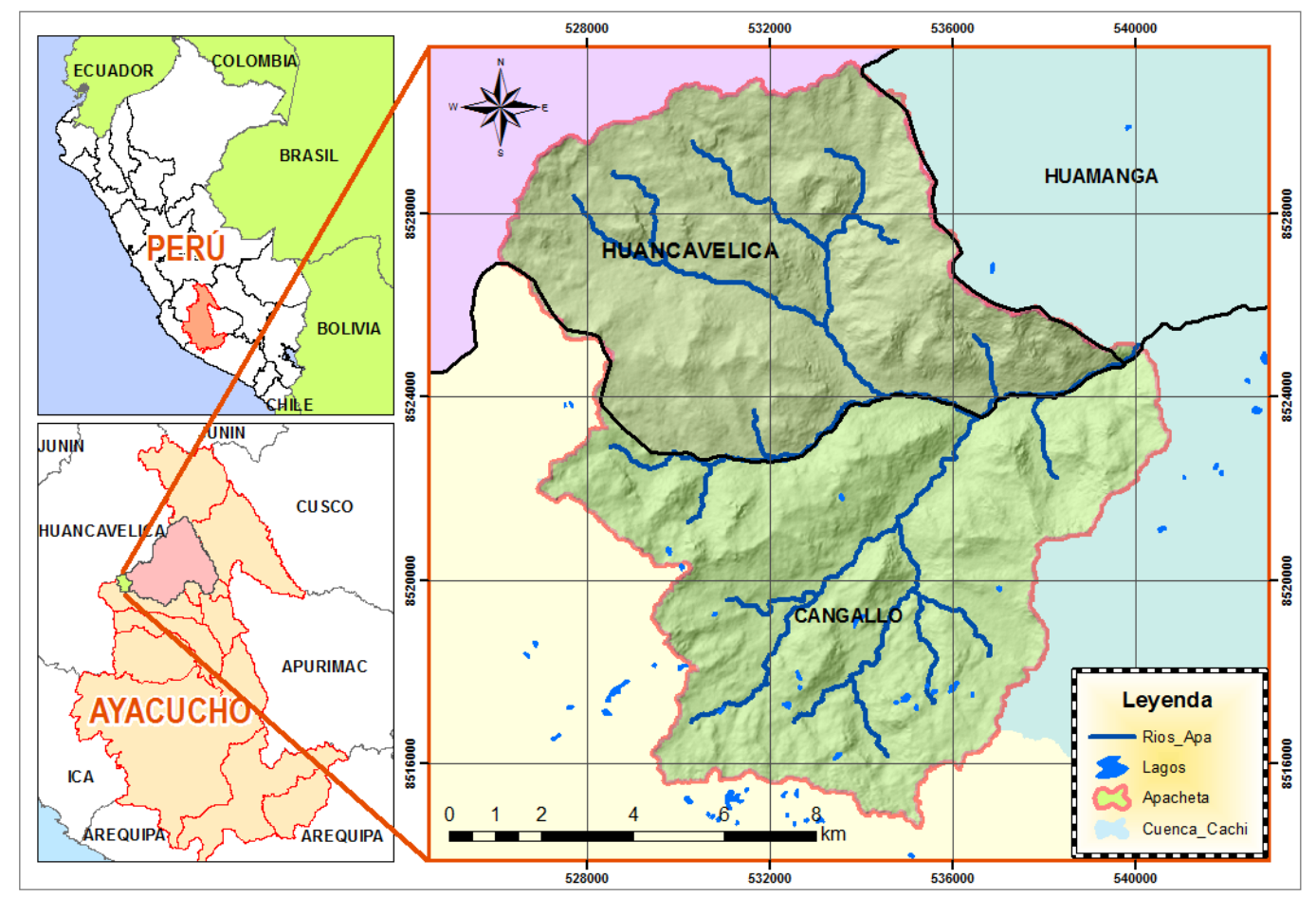

Figura 1: Ubicación geográfica de la microcuenca Apacheta, Región Ayacucho. 
Así se demostró en un experimento de absorción de agua, donde se obtuvo una correlación entre la humedad relativa y el contenido de agua en las partículas, estableciéndose una relación entre ambas para calcular las fuerzas de puente líquido. La mayor humedad mejoraría la ionización en las superficies de las partículas, lo que podría afectar las interacciones electrostáticas. Esto dio una idea clara del mecanismo de formación de neblina en la atmósfera, el mismo que es muy frecuente en zonas altoandinas como la microcuenca Apacheta ( $\mathrm{He}$ et al., 2019). La estacionalidad climática de la microcuenca Apacheta varía de manera directa con sus parámetros climáticos ya que las cuatro estaciones anuales no son diferenciadas en esta zona de altura entre los 4112 ms.n.m y los 5045 ms.n.m dando lugar a una variabilidad climática, por lo que para fundamentar la existencia de tres estadíos estacionales climáticos en la microcuenca Apacheta es necesario analizar los datos meteorológicos de ciertos parámetros climáticos como la precipitación, la temperatura máxima y mínima del aire, la velocidad del viento y la humedad relativa, en un periodo de datos comprendido entre los años 2000 al 2018.

\section{Material y Métodos}

La microcuenca Apacheta o cabecera de la cuenca Cachi se ubica en el distrito de Paras, provincia de Cangallo, Región Ayacucho, la zona norte se comparte con la provincia de Huaytará, Región Huancavelica, tal como se muestra en la Figura 1, tiene un área 14348.21 ha, su altitud mínima es de 4112 ms.n.m, su altitud máxima es de 5045 ms.n.m, y su altitud media es de 4651 ms.n.m. (Pereda et al., 2018).

La selección de grupos de precipitación según intensidad se realiza mediante la clasificación de intervalos de valores separables, donde la precipitación acumulada mensual se obtiene de las precipitaciones diarias, se calcula la media de todos los meses correspondientes al periodo comprendido entre los años 2000 al 2018, dando lugar a una media de cada mes del año, este método permite distinguir tres grupos de meses de precipitación muy significativos a los que se les ha denominado estadíos, el estadío lluvioso (color amarillo) que comprende los meses de enero, febrero, marzo y diciembre; el estadío intermedio (color verde) comprende los meses de abril, setiembre, octubre y noviembre; y el estadío seco (color rojo) comprende los meses de mayo, junio, julio y agosto. La estimación de los parámetros climáticos medidos en la estación meteorológica Apacheta, como la temperatura máxima y mínima del aire, la humedad relativa, la velocidad del viento y la radiación solar dentro de estos tres grupos respaldan la existencia de los tres estadíos estacionales en la microcuenca Apacheta, a partir de datos diarios desde el año 2000 al 2018.

\section{Resultados y Discusión}

\section{Precipitación en la microcuenca Apacheta}

La obtención de los tres grupos de estadíos estacionales se realizó mediante la selección de intervalos de valores de precipitación acumulada $(\mathrm{Pp})$ media mensual de todos los años desde el 2000 al 2018, distinguiéndose tres grupos significativos de cuatro meses cada uno, el estadío lluvioso comprendido en los meses de diciembre, enero, febrero y marzo, el estadío intermedio comprendido en los meses de abril, setiembre, octubre y noviembre, y el estadío seco comprendido en los meses de mayo, junio, julio y agosto. La tabla 1 , muestra los valores de la distribución estacional de la Pp media mensual, mínima y máxima en la microcuenca Apacheta, correspondientes a valores medios mensuales de Pp media mensual desde el año 2000 al 2018, donde se observa una Pp media anual de $71.09 \mathrm{~mm}$, la cual varía entre los valores de Pp mínima de $8.70 \mathrm{~mm}$ y Pp máxima de $139.65 \mathrm{~mm}$.

\begin{tabular}{lccr}
\hline Mes & PpMedia & PpMin & PpMax \\
\hline Enero & 148.03 & 7.91 & 277.66 \\
Febrero & 141.16 & 6.55 & 296.58 \\
Marzo & 143.32 & 7.70 & 203.89 \\
Abril & 54.46 & 3.12 & 141.01 \\
Mayo & 21.25 & 1.05 & 63.91 \\
Junio & 10.49 & 1.16 & 46.07 \\
Julio & 13.43 & 1.07 & 33.43 \\
Agosto & 16.47 & 2.31 & 68.80 \\
Setiembre & 39.92 & 0.44 & 98.01 \\
Octubre & 52.16 & 2.46 & 84.08 \\
Noviembre & 61.02 & 1.17 & 120.80 \\
Diciembre & 151.34 & 69.50 & 241.60 \\
Media & 71.09 & 8.70 & 139.65 \\
\hline
\end{tabular}

Tabla 1: Precipitación acumulada media mensual, mínima y máxima estacional ( $\mathrm{mm}$ ), en la microcuenca Apacheta, desde el año 2000 al 2018.

La figura 2, muestra el histograma de la distribución estacional de la precipitación acumulada media mensual (PpMedia), mínima (PpMin) y máxima (PpMax), se observa que la mayor intensidad de PpMedia se da en los meses de diciembre, enero, febrero y marzo corresspondiente al estadío lluvioso, donde la precipitación acumulada alcanza valores medios en los meses de diciembre de $151.34 \mathrm{~mm}$, en enero es de $148.03 \mathrm{~mm}$, en febrero es de $141.16 \mathrm{~mm}$ y en marzo es de $143.32 \mathrm{~mm}$. En los meses de mayo, junio, julio y agosto la PpMedia alcanza los valores más bajos en intensidad y corresponde al estadío seco, donde la PpMedia alcanza valores de 21.25 $\mathrm{mm}$ en el mes de mayo, $10.49 \mathrm{~mm}$ en junio, $13.43 \mathrm{~mm}$ en julio y $16.47 \mathrm{~mm}$ en agosto. En el estadío intermedio correspondiente a los meses de abril, setiembre, octubre y noviembre, la PpMedia alcanza valores de $54.46 \mathrm{~mm}$ 
en el mes de abril, $39.92 \mathrm{~mm}$ en setiembre, $52.16 \mathrm{~mm}$ en octubre y $61.02 \mathrm{~mm}$ en noviembre.

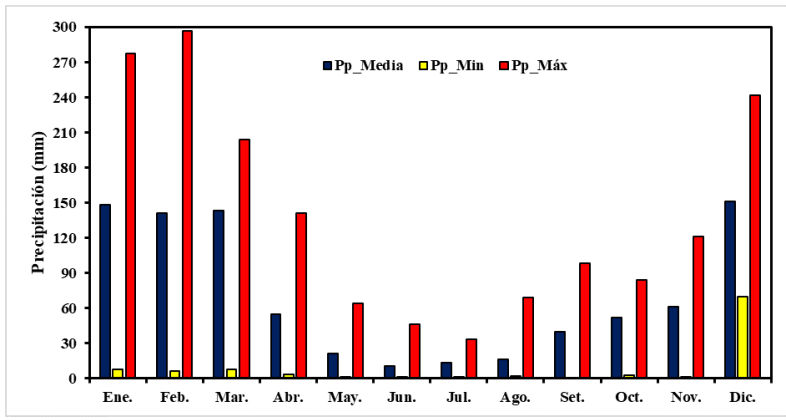

Figura 2: Distribución estacional de la Precipitación media mensual en la microcuenca Apacheta, 2000 al 2018.

La figura 3, muestra que el estadío lluvioso (color amarillo) mantiene una precipitación acumulada media anual de $145.96 \mathrm{~mm}$ el cual es mucho mayor que el estadío intermedio (color verde) que llega a alcanzar una precipitación acumulada media anual de $51.89 \mathrm{~mm}$ y que el estadío seco (color rojo) que llega alcanzar una precipitación acumulada media anual de $15.41 \mathrm{~mm}$. Se observa que el estadío lluvioso corresponde a los meses de lluvia y que el estadío seco corresponde a la temporada de frío en la zona altoandina.

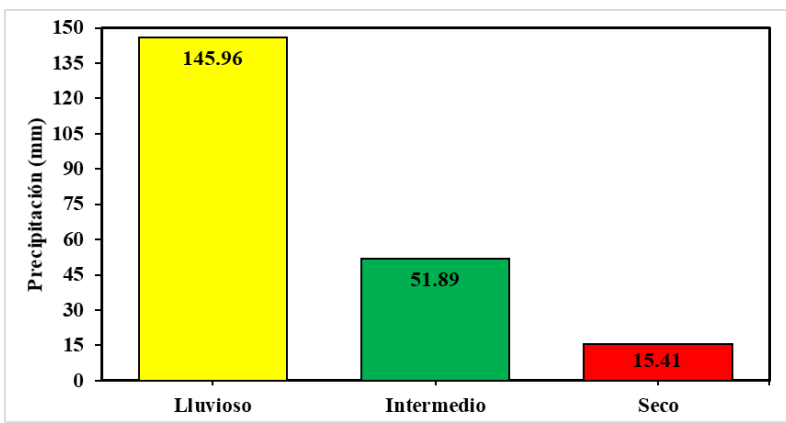

Figura 3: Estadíos de Precipitación en la microcuenca Apacheta. El estadío lluvioso (color amarillo). El estadío intermedio (color verde). El estadío seco (color rojo).

\section{Temperatura Máxima del aire en la micro- cuenca Apacheta}

La tabla 2, muestra los valores de la distribución estacional de la temperatura máxima (Tmx) media mensual del aire, comprendida entre los valores mínima y máxima, que corresponden a valores medios mensuales de la Tmx desde el año 2000 al 2018. Se observa que la estacionalidad de la Tmx mantiene una clara correspondencia con los tres estadíos: lluvioso, intermedio y seco, de donde se obtiene que la Tmx del aire media mensual representativa es $12.71^{\circ} \mathrm{C}$ en la microcuenca Apacheta, la cual varía entre los valores de la Tmx mínima de $10.86{ }^{\circ} \mathrm{C}$ y la $\mathrm{Tmx}$ máxima de $14.30{ }^{\circ} \mathrm{C}$.

\begin{tabular}{lccr}
\hline Mes & TmxMedia & TmxMin & TmxMax \\
\hline Enero & 12.29 & 10.61 & 14.94 \\
Febrero & 12.09 & 10.71 & 13.34 \\
Marzo & 11.90 & 10.43 & 14.29 \\
Abril & 12.18 & 11.30 & 13.56 \\
Mayo & 12.69 & 11.80 & 13.88 \\
Junio & 12.41 & 11.07 & 13.55 \\
Julio & 12.14 & 10.21 & 13.83 \\
Agosto & 12.78 & 11.14 & 14.42 \\
Setiembre & 13.05 & 10.85 & 14.52 \\
Octubre & 13.63 & 11.70 & 14.84 \\
Noviembre & 14.66 & 13.10 & 15.80 \\
Diciembre & 12.91 & 11.35 & 14.66 \\
Media & 12.73 & 11.19 & 14.30 \\
\hline
\end{tabular}

Tabla 2: Temperatura Máxima media mensual del aire, mínima y máxima estacional $\left({ }^{\circ} \mathrm{C}\right)$, en la microcuenca Apacheta, desde el año 2000 al 2018.

La figura 4, muestra la distribución estacional de la temperatura máxima media mensual del aire (TmxMedia), mínima (TmxMin) y máxima (TmxMax) y su comportamiento con los tres estadíos del año, se observa que la TmxMedia empieza a incrementarse a partir de los meses de setiembre, octubre, alcanzando el valor más alto durante el mes de noviembre. En los meses correspondientes al estadío lluvioso la TmxMedia llega a alcanzar valores medios característicos en los meses de diciembre de $12.71{ }^{\circ} \mathrm{C}$, en enero es de $12.29{ }^{\circ} \mathrm{C}$, en febrero es de $12.09{ }^{\circ} \mathrm{C}$ y en marzo es de $11.98{ }^{\circ} \mathrm{C}$.

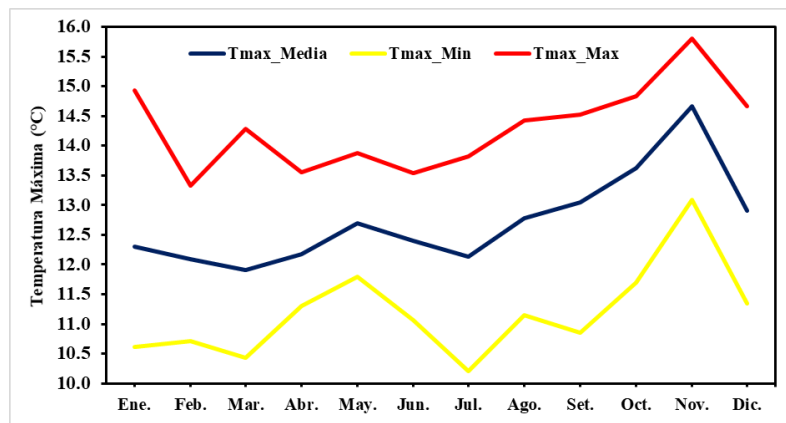

Figura 4: Distribución estacional de la Temperatura Máxima media mensual del aire en la microcuenca Apacheta, dentro del rango de temperatura máxima mínima y temperatura máxima máxima del aire.

Durante el estadío seco la temperatura máxima alcanza valores medios en los meses de mayo de $12.69{ }^{\circ} \mathrm{C}$, en junio es de $12.41{ }^{\circ} \mathrm{C}$, en julio es de $12.14^{\circ} \mathrm{C}$ y en agosto es de $12.78{ }^{\circ} \mathrm{C}$. En el estadío intermedio la temperatura máxima alcanza valores medios en los meses de abril de $12.18^{\circ} \mathrm{C}$, en setiembre es de $13.05^{\circ} \mathrm{C}$, en octubre es de 
$13.63{ }^{\circ} \mathrm{C}$ y en noviembre es de $14.66{ }^{\circ} \mathrm{C}$, en donde se observa que el mes de noviembre es el mes con el mayor valor y los meses de marzo y julio los meses con menores valores comparados con el resto de meses de todo el año.

La figura 5, muestra que la temperatura máxima media anual del aire en el estadío lluvioso (amarillo) es de $12.25{ }^{\circ} \mathrm{C}$, en el estadío intermedio (verde) es de $13.38{ }^{\circ} \mathrm{C}$ y en el estadío seco (rojo) es de $12.51{ }^{\circ} \mathrm{C}$. lo cual signifca que la temperatura máxima de aire más alta comparadas se da durante el estadío intermedio que no precisamente es verano, esto posiblemente a que en estos meses del año el encapotamiento de nubes es menor durante la mayor parte del dia, al contrario del estadío lluvioso que si presenta un alto grado de encapotamiento de nubes durante la mayor parte del dia.

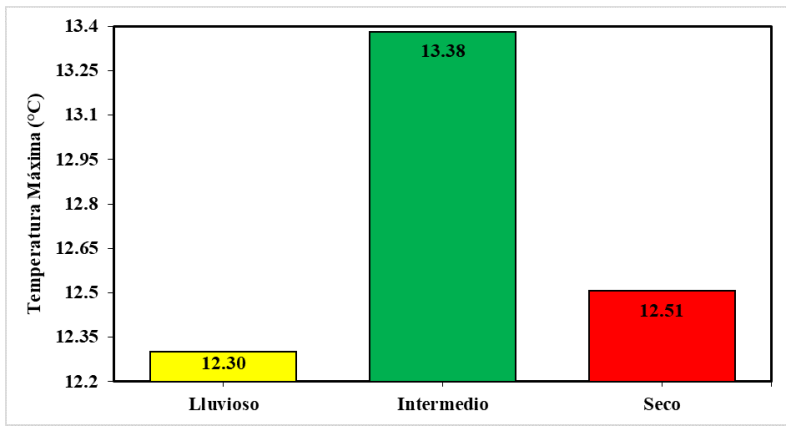

Figura 5: Estadíos de la Temperatura Máxima del aire en la microcuenca Apacheta.

\begin{tabular}{lccr}
\hline Mes & TmnMedia & TmnMin & TmnMax \\
\hline Enero & 1.52 & -1.59 & 3.00 \\
Febrero & 1.88 & -0.65 & 3.62 \\
Marzo & 2.04 & -0.14 & 3.37 \\
Abril & 0.77 & -1.83 & 2.40 \\
Mayo & -1.31 & -3.13 & 0.65 \\
Junio & -2.49 & -4.69 & -0.59 \\
Julio & -3.06 & -6.20 & -1.43 \\
Agosto & -2.75 & -5.34 & -0.91 \\
Setiembre & -1.56 & -4.23 & 0.42 \\
Octubre & -0.23 & -2.97 & 1.98 \\
Noviembre & 0.23 & -2.21 & 2.36 \\
Diciembre & 0.78 & -2.67 & 2.18 \\
Media & -0.35 & -2.97 & 1.42 \\
\hline
\end{tabular}

Tabla 3: Temperatura Mínima media mensual del aire, mínima y máxima estacional $\left({ }^{\circ} \mathrm{C}\right)$, en la microcuenca Apacheta, desde el año 2000 al 2018.

\section{Temperatura Mínima del aire en la micro- cuenca Apacheta}

La tabla 3, muestra los valores de la distribución estacional de la temperatura mínima (Tmn) media mensual del aire, mínima y máxima, estos valores corresponden a los valores medios mensuales de la Tmn desde el año 2000 al 2018, se observa que los valores de la Tmn del aire se distribuyen de manera estacional para los tres estadíos lluvioso, intermedio y seco, llegando a alcanzar una Tmn media mensual del aire media anual de -0.35 ${ }^{\circ} \mathrm{C}$, que fluctúa entre el valor Tmn mínimo de $-2.97{ }^{\circ} \mathrm{C}$ y Tmn máximo de $1.42{ }^{\circ} \mathrm{C}$.

La figura 6, muestra la distribución estacional de la temperatura mínima media mensual del aire (TmnMedia), mínima (TmnMin) y máxima (TmnMax) y su comportamiento entre los distintos estadíos del año, se observa que la TmnMedia empieza a disminuir a partir de los meses de mayo, junio, alcanzando los valores más bajos de la TmnMedia durante los meses de junio, julio y agosto correspondientes al estadío seco, siendo el valor más bajo alcanzado en el mes de julio que coincide con los meses de frio en invierno. Durante el estadío lluvioso la TmnMedia llega a tener valores medios en los meses de diciembre de $0.78{ }^{\circ} \mathrm{C}$, en enero es de $1.52{ }^{\circ} \mathrm{C}$, en febrero es de $1.88{ }^{\circ} \mathrm{C}$ y en marzo es de $2.04{ }^{\circ} \mathrm{C}$, siendo el valor más alto alcanzado en el mes de febrero. Durante el estadío seco la temperatura mínima del aire alcanza valores medios en los meses de mayo de $-1.31^{\circ} \mathrm{C}$, en junio es de $2.49{ }^{\circ} \mathrm{C}$, en julio es de $-3.06{ }^{\circ} \mathrm{C}$ y en agosto es de -2.75 ${ }^{\circ} \mathrm{C}$. En el estadío intermedio la temperatura mínima del aire alcanza valores medios en los meses de abril de 0.77 ${ }^{\circ} \mathrm{C}$, en setiembre es de $-1.56{ }^{\circ} \mathrm{C}$, en octubre es de $-0.23{ }^{\circ} \mathrm{C}$ y en noviembre sube a $0.23{ }^{\circ} \mathrm{C}$.

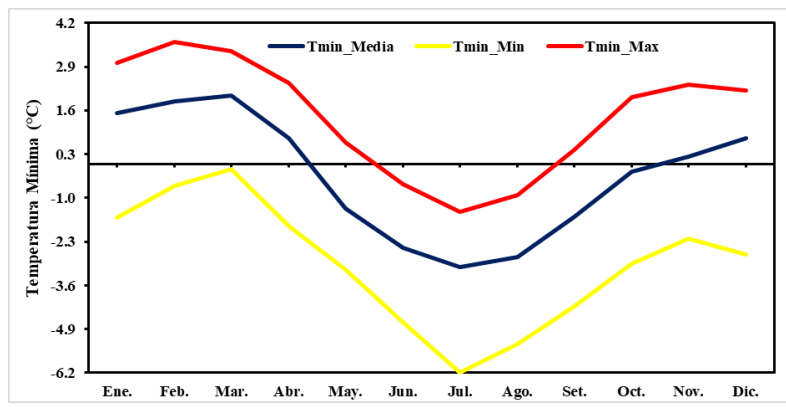

Figura 6: Distribución estacional de la Temperatura Mínima media mensual del aire en la microcuenca Apacheta, dentro del rango de temperatura mínima mínima y temperatura mínima máxima del aire.

La figura 7, muestra que la temperatura mínima media anual del aire para el estadío lluvioso (amarillo) es de $1.55{ }^{\circ} \mathrm{C}$, para el estadío intermedio (verde) es de -0.2 ${ }^{\circ} \mathrm{C}$ y para el estadío seco (rojo) es de $-2.4{ }^{\circ} \mathrm{C}$. Además, se observa que la temperatura mínima del aire muestra su valor más alto durante el estadío lluvioso y la temperatura mínima del aire más baja se presenta durante el estadío seco, que coincidentemente corresponde a los meses de frío o invierno, lo cual fortalece la existencia de los tres estadíos en la microcuenca Apacheta. 


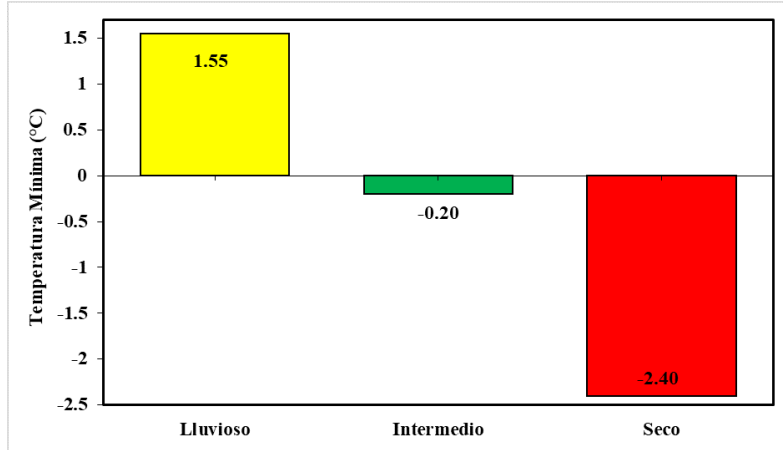

Figura 7: Estadíos de la Temperatura Mínima del aire en la microcuenca Apacheta.

\section{Humedad Relativa en la microcuenca Apa- cheta}

La tabla 4, muestra los valores de la distribución estacional de la humedad relativa (HR) media mensual, mínima y máxima, estos valores corresponden a los valores medios mensuales de la HR desde el año 2000 al 2018, donde se observa que los valores de la HR media anual es $70.43 \%$, la cual fluctúa entre el valor de la HR mínimo de $66.25 \%$ y HR máximo de $74.17 \%$.

\begin{tabular}{lccr}
\hline Mes & HRMedia & HRMin & HRMax \\
\hline Enero & 73.95 & 70 & 79 \\
Febrero & 74.79 & 70 & 78 \\
Marzo & 75.58 & 71 & 79 \\
Abril & 72.74 & 68 & 75 \\
Mayo & 68.74 & 66 & 73 \\
Junio & 67.37 & 64 & 71 \\
Julio & 66.89 & 63 & 70 \\
Agosto & 66.94 & 64 & 71 \\
Setiembre & 68.32 & 64 & 73 \\
Octubre & 69.22 & 65 & 73 \\
Noviembre & 68.78 & 65 & 73 \\
Diciembre & 71.84 & 65 & 75 \\
Media & 70.43 & 66.25 & 74.17 \\
\hline
\end{tabular}

Tabla 4: Humedad Relativa media mensual, mínima y máxima estacional (\%), en la microcuenca Apacheta, 2000 al 2018.

La figura 8, muestra la distribución estacional de la humedad relativa media mensual (HRMedia), fluctuando entre los valores mínimo (HRMin) y máximo (HRMax) y su comportamiento entre los distintos estadíos del año, se observa que en el estadío lluvioso la humedad relativa llega a tener valores medios en los meses de diciembre de $71.84 \%$, en enero es de $73.95 \%$, en febrero es de $74.79 \%$ y en el mes de marzo es de $75.58 \%$. Durante el estadío seco la humedad relativa alcanza valores medios en los meses de mayo de $68.74 \%$, en el mes de junio de $67.36 \%$, en el mes de julio de $66.89 \%$ y en el mes de agosto de $66.94 \%$. En el estadío intermedio la humedad relativa alcanza valores medios en los meses de abril de $72.74 \%$, en setiembre es de $68.32 \%$, en octubre es de $69.22 \%$ y en el mes de noviembre es de $68.78 \%$.

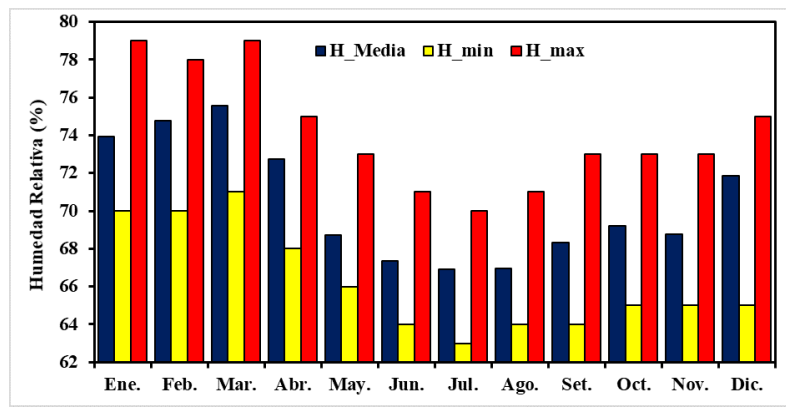

Figura 8: Distribución estacional de la Humedad Relativa Media mensual, mínima y máxima, microcuenca Apacheta.

La figura 9, muestra los estadíos de la humedad relativa media anual en donde se observa que la humedad relativa más alta se da durante el estadío lluvioso con un porcentaje de $74.04 \%$ esto debido a la alta presencia de precipitación durante esos meses y la humedad relativa más baja en el estadío seco con un porcentaje de $67.49 \%$, que coincidentemente corresponde con los meses de frío en Apacheta. Durante el estadío intermedio el porcentaje de humedad relativa media es de $69.76 \%$, que precisamente es mayor que en el estadío seco y menor que el estadío lluvioso.

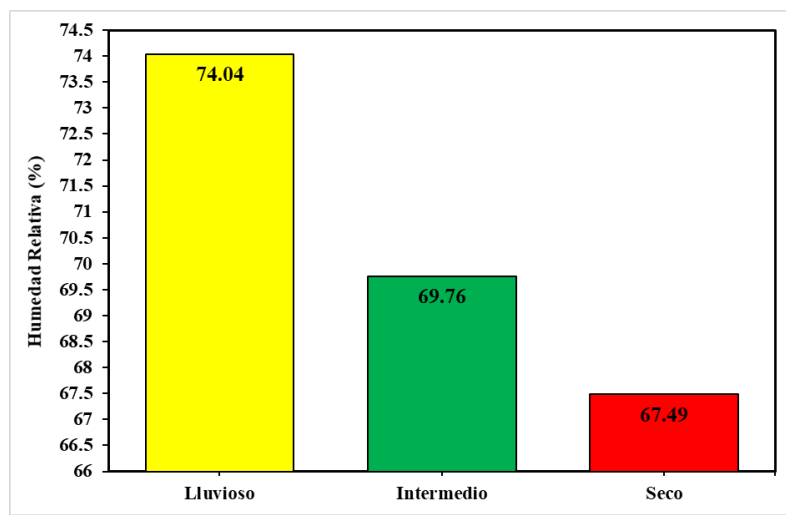

Figura 9: Estadíos de la Humedad Relativa en la microcuenca Apacheta.

\section{Velocidad del viento en la microcuenca Apacheta}

La tabla 5, muestra la estacionalidad de la velocidad del viento $(\mathrm{Vv})$ media mensual, mínima y máxima, estos valores corresponden a los valores medios mensuales de la Vv desde el 2000 al 2018, donde se obtiene un valor representativo de la $\mathrm{Vv}$ media anual de $2.91 \mathrm{~m} / \mathrm{s}$, que 
varía entre el valor de Vv mínimo de $1.79 \mathrm{~m} / \mathrm{s}$ y un valor máximo de $\mathrm{Vv}_{\mathrm{v}}$ de $5.64 \mathrm{~m} / \mathrm{s}$.

\begin{tabular}{lccr}
\hline Mes & VvMedia & VvMin & VvMax \\
\hline Enero & 2.54 & 1.58 & 4.57 \\
Febrero & 2.54 & 1.52 & 4.59 \\
Marzo & 2.52 & 1.65 & 4.93 \\
Abril & 2.68 & 1.76 & 5.18 \\
Mayo & 3.09 & 1.97 & 5.76 \\
Junio & 3.16 & 2.00 & 6.11 \\
Julio & 3.15 & 2.02 & 6.24 \\
Agosto & 3.23 & 1.86 & 6.39 \\
Setiembre & 3.13 & 1.77 & 6.12 \\
Octubre & 3.02 & 2.02 & 5.36 \\
Noviembre & 3.02 & 1.82 & 6.58 \\
Diciembre & 2.79 & 1.62 & 5.81 \\
Media & 2.91 & 1.79 & 5.64 \\
\hline
\end{tabular}

Tabla 5: Velocidad del viento media mensual, mínima y máxima estacional, en la microcuenca Apacheta $(\mathrm{m} / \mathrm{s})$, desde el año 2000 al 2018.

La figura 10, muestra la distribución estacional de la velocidad media mensual del viento, mínima y máxima, se observa que en el estadío lluvioso la velocidad del viento llega a tener valores medios en los meses de diciembre de $2.8 \mathrm{~m} / \mathrm{s}$, en enero de $2.54 \mathrm{~m} / \mathrm{s}$, en febrero de $2.54 \mathrm{~m} / \mathrm{s}$ y en marzo de $2.52 \mathrm{~m} / \mathrm{s}$. Durante el estadío seco la temperatura mínima alcanza valores medios en los meses de mayo de $3.09 \mathrm{~m} / \mathrm{s}$, en junio de $3.16 \mathrm{~m} / \mathrm{s}$, en julio de 3.15 $\mathrm{m} / \mathrm{s}$ y en agosto de $3.23 \mathrm{~m} / \mathrm{s}$. En el estadío intermedio la temperatura mínima alcanza valores medios en los meses de abril de $2.68 \mathrm{~m} / \mathrm{s}$, en setiembre $3.13 \mathrm{~m} / \mathrm{s}$, en octubre de $3.02 \mathrm{~m} / \mathrm{s}$ y en noviembre de $3.02 \mathrm{~m} / \mathrm{s}$.

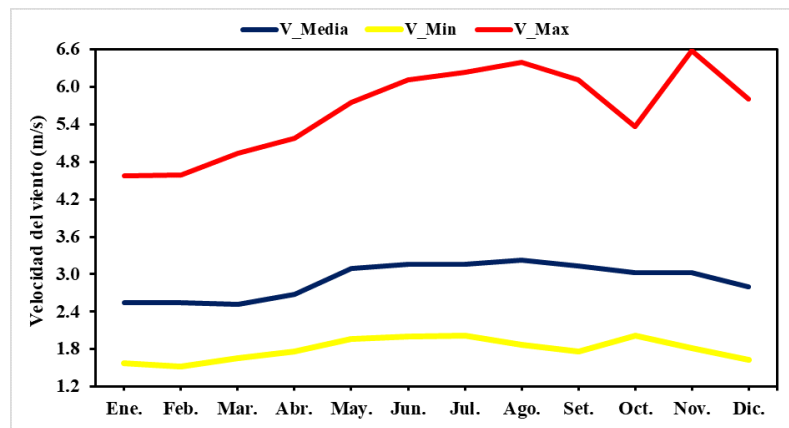

Figura 10: Distribución estacional de la velocidad del viento en la microcuenca Apacheta.

La figura 11, muestra los estadíos de la velocidad del viento media anual en donde se observa que la velocidad media del viento más baja se da durante el estadío lluvioso con un valor de $2.6 \mathrm{~m} / \mathrm{s}$ y la velocidad media del viento más alta se da en el estadío seco con un valor de $3.16 \mathrm{~m} / \mathrm{s}$ tal vez por la baja humedad relativa o coincidentemente corresponde con los meses de frío o invierno en Apacheta. Durante el estadío intermedio la velocidad media del viento es de $2.96 \mathrm{~m} / \mathrm{s}$ que es mayor en el estadío lluvioso y menor que en el estadío seco.

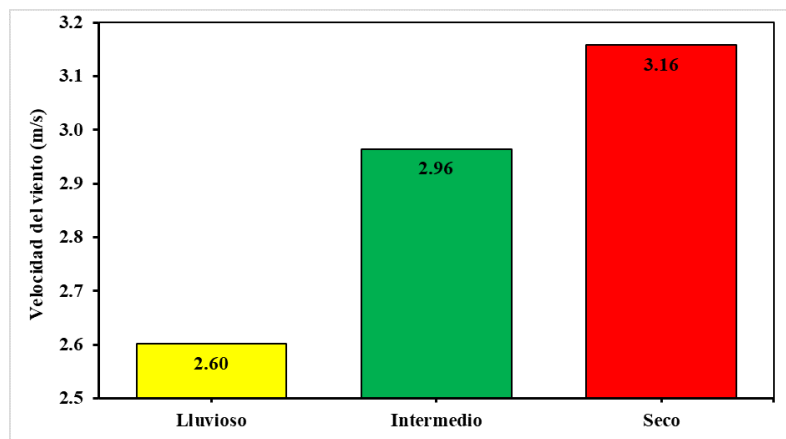

Figura 11: Estadíos de la velocidad del viento en la microcuenca Apacheta.

\section{Radiación Solar en la microcuenca Apache- ta}

La tabla 6, muestra los valores de la radiación solar media mensual (RS), mínima y máxima estacional en la microcuenca Apacheta, estos valores corresponden a los valores medios mensuales de la RS desde el año 2000 al 2018, donde se obtiene un valor representativo para la RS media anual de $514.43 \mathrm{Ly}$, la cual varía entre los valores mínimo de RS de 461.20 Ly y máximo de RS de 573.54 Ly, en unidades de Langley (Ly), donde $1 \mathrm{MJ} / \mathrm{m}^{2}$ $=23.9234 \mathrm{Ly}$.

\begin{tabular}{lccr}
\hline Mes & RSMedia & RSMin & RSMax \\
\hline Enero & 539.16 & 459.33 & 595.69 \\
Febrero & 518.78 & 468.90 & 588.52 \\
Marzo & 480.06 & 430.62 & 538.59 \\
Abril & 466.59 & 433.01 & 533.49 \\
Mayo & 456.82 & 410.87 & 488.04 \\
Junio & 436.73 & 390.72 & 473.68 \\
Julio & 454.46 & 413.87 & 507.18 \\
Agosto & 507.15 & 455.32 & 547.85 \\
Setiembre & 548.81 & 483.25 & 614.21 \\
Octubre & 582.27 & 523.92 & 643.54 \\
Noviembre & 611.78 & 538.28 & 667.46 \\
Diciembre & 570.54 & 526.31 & 684.21 \\
Media & 514.43 & 461.20 & 573.54 \\
\hline
\end{tabular}

Tabla 6: Radiación Solar media mensual, mínima y máxima estacional, en la microcuenca Apacheta, 2000 al 2018.

La figura 12, muestra la distribución estacional de la radiación solar media mensual, mínima y máxima y su comportamiento entre los tres estadíos del año, se observa que para los valores más altos durante el año la 
RS empieza a incrementarse a partir de los meses de setiembre, octubre, alcanzando los más altos picos en los meses de noviembre, diciembre, y empezando a decrecer durante los meses de enero y febrero.

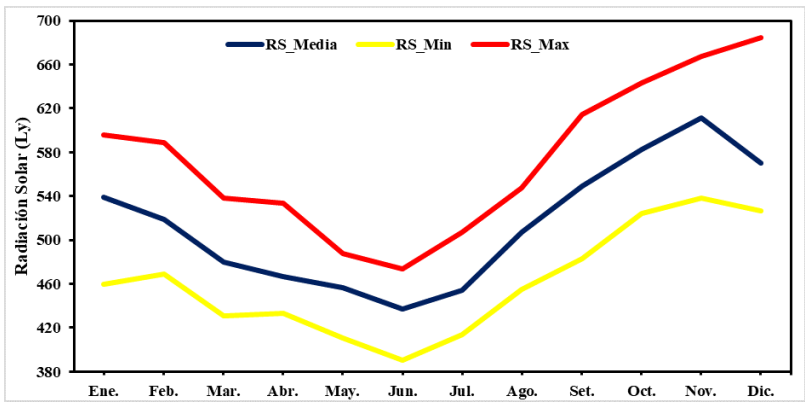

Figura 12: Distribución estacional de la radiación solar media mensual, mínima y máxima en la microcuenca Apacheta.

El decrecimiento de la RS se da a partir de los meses de marzo, abril, mayo, siendo el mes de junio el mes con el más bajo valor de RS, para luego empezar a aumentar durante los meses de julio, agosto. En el estadío lluvioso la radiación solar llega a tener valores medios en los meses de diciembre de $570.54 \mathrm{Ly}$, en enero de 539.16 Ly, en febrero de 518.78 Ly y en marzo de 480.06 Ly. Durante el estadío seco la radiación solar alcanza valores medios en los meses de mayo de $456.82 \mathrm{Ly}$, en junio de $436.73 \mathrm{Ly}$, en julio de 454.46 Ly y en agosto de 507.15 Ly. Así mismo, en el estadío intermedio la radiación solar alcanza valores medios en los meses de abril de $466.6 \mathrm{Ly}$, en setiembre $548.81 \mathrm{Ly}$, en octubre de 582.27 Ly y en noviembre de $611.78 \mathrm{Ly}$.

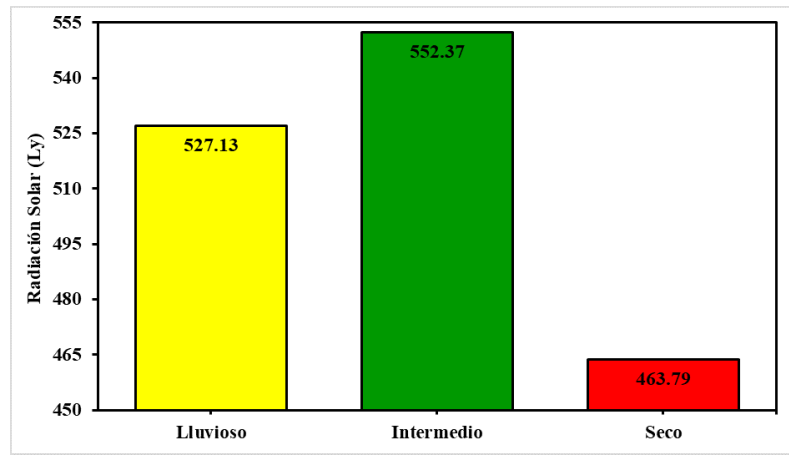

Figura 13: Estadíos de la Radiación Solar en la microcuenca Apacheta.

La figura 13, muestra que durante el estadío seco la radiación solar media anual es de 463.79 Ly y corresponde al estadío con la radiación solar más baja durante todo el año en la microcuenca Apacheta, que coincidentemente corresponde a los meses de frío o invierno en la microcuenca Apacheta. La radiación solar más alta de 552.37 Ly se presenta durante el estadío intermedio, es decir, en los meses de abril, setiembre, octubre y noviembre, donde hay precipitaciones intermedias y el encapotamiento de nubes es menor en comparación con el resto de estadíos, mostrandose un cielo de Apacheta libre de nubes en la mayor parte del día. Durante el estadío lluvioso se presenta una radiación solar media de 527.13 Ly que es menor que el estadío lluvioso y mayor que el estadío seco, esto posiblemente por el alto grado de encapotamiento de nubes durante la mayor parte del dia.

\section{Conclusiones}

1. Se ha logrado determinar la existencia de tres grupos notables de estadíos estacionales climáticos en la microcuenca Apacheta, el estadío lluvioso, intermedio y seco, a partir de la evaluación de la precipitación acumulada mensual. Estos estadios se fundamentan mediante el comportamiento estacional de la temperatura máxima y mínima del aire, la velocidad del viento, la humedad relativa y radiación solar, de donde se observa que el estadío lluvioso presenta un registro aproximado de precipitación media acumulada de $145.96 \mathrm{~mm}$ con influencia de la temperatura máxima del aire de $12.3^{\circ} \mathrm{C}$, con temperatura mínima del aire de $1.55{ }^{\circ} \mathrm{C}$, humedad relativa de $74.04 \%$, velocidad del viento de $2.6 \mathrm{~m} / \mathrm{s}$ y radiación solar de 527.13 Ly. El estadío intermedio presenta un registro aproximado de precipitación de 51.89 $\mathrm{mm}$ con una temperatura máxima del aire de $13.38{ }^{\circ} \mathrm{C}$, con temperatura mínima del aire de $-0.2{ }^{\circ} \mathrm{C}$, humedad relativa de $69.76 \%$, velocidad del viento de $2.96 \mathrm{~m} / \mathrm{s}$ y radiación solar de 552.37 Ly. El estadío seco presenta un registro aproximado de precipitación de $15.41 \mathrm{~mm}$ con una temperatura máxima del aire de $12.51{ }^{\circ} \mathrm{C}$, con temperatura mínima del aire de $-2.4{ }^{\circ} \mathrm{C}$, humedad relativa de $67.49 \%$, velocidad del viento de $3.16 \mathrm{~m} / \mathrm{s}$ y radiación solar de 463.79 Ly en la microcuenca Apacheta.

2. Comparando las características climáticas de los tres estadíos, se verifica que el comportamiento de la precipitación en el estadío lluvioso es más alto comparado con los otros dos estadíos, por lo que en este estadío la temperatura máxima del aire es menor en comparación con los otros dos estadíos, la temperatura mínima del aire es la más alta, la humedad relativa es la más alta, la velocidad del aire es menor y la radiación solar mantiene un valor moderado situado entre el estadío intermedio que es el más alto y el estadío seco que es el más bajo. En el estadío intermedio de precipitación se tiene que la temperatura máxima del aire mantiene un valor mayor comparado con los otros dos estadíos al igual que la radiación solar, la temperatura mínima del aire mantiene valores moderados entre los otros dos estadíos, al igual que la humedad relativa y la velocidad del viento. Durante el estadio seco de precipitaciones muy bajas la temperatura mínima del aire, la humedad relativa y la 
radiación solar muestran valores muy bajos comparados con los otros dos estadíos.

\section{Agradecimientos}

Este trabajo ha sido posible gracias al apoyo financiero de los proyectos "Strengthening resilience of
Andean river basin headwaters facing global change" (PGA084063), financiado por el Programa PEER de USAID e "International Water Security Network", financiado por el Lloyd's Register Foundation. Los autores agradecen a OPEMAN del Gobierno Regional de Ayacucho por facilitar los datos hidro meteorológicos y a la Universidad Nacional de San Cristóbal de Huamanga por su incondicional apoyo.

\section{Referencias}

Aguilera, J., Victoria de Gálvez, M., Conde, R., PérezRodríguez, E., Viñegla, B., Abdala, R., Segovia, M., Herrera, E., and Figueroa, F. L. (2004). Series temporales de medida de radiación solar ultravioleta y fotosintética en málaga. Actas Dermo-Sifiliográficas, $95(1): 25-31$.

Campos, F. (2015). Búsqueda de tendencias en la precipitación anual del estado de zacatecas, méxico; en 30 registros con más de 50 años. Ingeniería, Investigación y Tecnología, 16(3):355-368.

He, Y., Gu, Z., Lu, W., Zhang, L., Okuda, T., Fujioka, K., Luo, H., and Yu, C. W. (2019). Atmospheric hu- midity and particle charging state on agglomeration of aerosol particles. 197:141-149.

Moncada, W., Pereda, A., Aldana, C., Masias, M., and Jimenez, J. (2015). Cuantificación hidrográfica de la cuenca del rio cachi-ayacucho, mediante imágenes satelitales. Instituto de Investigación Científica e innovación Tecnológica de la UNSCH, II(2):40-46.

Pereda, A., Moncada, W., and Verde, L. (2018). Respuesta nival de la cabecera de cuenca Cachi-Apacheta de Ayacucho, volume I. Editorial Académica Española.

Pérez, E., Ramírez, V., and Peña, A. (2016). Variabilidad espacial y temporal de la temperatura del aire en la zona cafetera colombiana. Investigaciones Geográficas, Boletín del Instituto de Geografía, 2016(89):23-40. 